Proc. Indian Acad. Sci. (Math. Sci.) Vol. 116, No. 2, May 2003, pp. 233-255.

Printed in India

\title{
Multiplicity of nontrivial solutions for elliptic equations with nonsmooth potential and resonance at higher eigenvalues
}

\author{
LESZEK GASIŃSKI $^{1}$, DUMITRU MOTREANU ${ }^{2}$ \\ and NIKOLAOS S PAPAGEORGIOU ${ }^{3}$ \\ ${ }^{1}$ Institute of Computer Science, Jagiellonian University, ul. Nawojki 11, \\ 30072 Cracow, Poland \\ ${ }^{2}$ Département de Mathematiques, Université de Perpignan, 66860 Perpignan, France \\ ${ }^{3}$ Department of Mathematics, National Technical University, Zografou Campus, \\ Athens 15780, Greece \\ E-mail:npapg@math.ntua.gr
}

MS received 15 March 2004; revised 22 February 2005

\begin{abstract}
We consider a semilinear elliptic equation with a nonsmooth, locally Lipschitz potential function (hemivariational inequality). Our hypotheses permit double resonance at infinity and at zero (double-double resonance situation). Our approach is based on the nonsmooth critical point theory for locally Lipschitz functionals and uses an abstract multiplicity result under local linking and an extension of the Castro-LazerThews reduction method to a nonsmooth setting, which we develop here using tools from nonsmooth analysis.
\end{abstract}

Keywords. Double resonance; reduction method; eigenvalue; hemivariational inequality; locally Lipschitz function; Clarke subdifferential; critical point; local linking; nonsmooth Cerami condition.

\section{Introduction}

Let $Z \subseteq \mathbb{R}^{N}$ be a bounded domain with a $C^{2}$ boundary $\Gamma$. We study the following resonant semilinear elliptic differential equation with a nonsmooth potential (hemivariational inequality):

$$
\left\{\begin{array}{l}
-\Delta x(z)-\lambda_{k} x(z) \in \partial j(z, x(z)), \text { for a.a. } z \in Z \\
\left.x\right|_{\Gamma}=0 .
\end{array}\right.
$$

Here $k \geq 1$ is a fixed integer, $\left\{\lambda_{n}\right\}_{n \geq 1}$ is the increasing sequence of distinct eigenvalues of the negative Laplacian with Dirichlet boundary condition (i.e. of $\left.\left(-\Delta, H_{0}^{1}(Z)\right)\right), j(z, \zeta)$ is a locally Lipschitz in the $\zeta$-variable integrand (in general it can be nonsmooth) and $\partial j(z, \zeta)$ is the Clarke subdifferential with respect to the $\zeta$-variable.

For problem (HVI), we prove a multiplicity result, using a recent abstract theorem on the existence of multiple nontrivial critical points for a nonsmooth locally Lipschitz functional, proved by Kandilakis, Kourogenis and Papageorgiou [21]. Our approach is variational and is based on the nonsmooth critical point theory (see [9] and [22]). In particular, we develop and use a nonsmooth variant of the so-called 'reduction method'. This method was first introduced for smooth problems by Castro and Lazer [7] and Thews [36] (see 
also [56]). Our hypotheses allow the nonsmooth potential to interact asymptotically at $\pm \infty$ with two consecutive eigenvalues of higher order. Berestycki and de Figueiredo [2] were the first to consider such problems with resonance between $\lambda_{1}$ and $\lambda_{2}$ and they coined the term 'double resonance problems'. In their analysis, the use of the interval $\left[\lambda_{1}, \lambda_{2}\right]$ is crucial, since they exploit heavily the fact that the principal eigenfunction $u_{1}$ is strictly positive and $\partial u_{1} / \partial n<0$ with $n$ being the outward unit normal on the boundary (this is a consequence of the strong maximum principle). It is well-known that in higher parts of the spectrum this is no longer true. Recall that the principal eigenfunction $u_{1}$ is the only one with constant sign. So in higher parts of the spectrum the analysis is more delicate. Recently for smooth problems, the issue was investigated by Cac [4], Hirano and Nishimura [16], Robinson [31], Costa-Silva [11], Landesman, Robinson and Rumbos [23], Iannacci and Nkashama [19], Tang and $\mathrm{Wu}[34], \mathrm{Su}$ and Tang [33] and Su [32].

For problems with nonsmooth potential (known in the literature as hemivariational inequalities), equations resonant at higher eigenvalues were investigated by Goeleven, Motreanu and Panagiotopoulos [15] and Gasiński and Papageorgiou [14]. However, they did not allow for the situation of double resonance.

We should mention that hemivariational inequalities arise in physical problems, when one wants to consider more realistic models with a nonsmooth and nonconvex energy functionals. For concrete applications we refer to the book of Naniewicz and Panagiotopoulos [27]. For the mathematical theory of hemivariational inequalities we refer to the work of Gasiński and Papageorgiou [12 13], Motreanu and Panagiotopoulos [25 26], Niculescu and Radulescu [28], Radulescu [29], Radulescu and Panagiotopou$\operatorname{los}[30]$ and the references therein.

\section{Mathematical background}

As we have already mentioned, our approach is based on the theory of the nonsmooth critical point theory for locally Lipschitz functionals.

Let $X$ be a Banach space and $X^{*}$ its topological dual. By $\|\cdot\|_{X}$ we denote the norm of $X$ and by $\langle\cdot, \cdot\rangle_{X}$ the duality pairing for the pair $\left(X, X^{*}\right)$.

We will be dealing with locally Lipschitz functions $\varphi: X \rightarrow \mathbb{R}$.

Recall that a continuous convex function is locally Lipschitz. For a locally Lipschitz function $\varphi: X \rightarrow \mathbb{R}$, we introduce the generalized directional derivative of $\varphi$ at $x \in X$ in the direction $h \in X$, defined by

$$
\varphi^{0}(x ; h) \stackrel{d f}{=} \limsup _{\substack{x^{\prime} \rightarrow x \\ t \searrow 0}} \frac{\varphi\left(x^{\prime}+t h\right)-\varphi\left(x^{\prime}\right)}{t}
$$

(see [10]). It is easy to check that the function $X \ni h \rightarrow \varphi^{0}(x ; h) \in \mathbb{R}$ is sublinear, continuous, and so by the Hahn-Banach theorem, $\varphi^{0}(x ; \cdot)$ is the support function of a nonempty, convex and $w^{*}$-compact set $\partial \varphi(x)$, defined by

$$
\partial \varphi(x) \stackrel{d f}{=}\left\{x^{*} \in X^{*}:\left\langle x^{*}, h\right\rangle_{X} \leq \varphi^{0}(x ; h) \text { for all } h \in X\right\} .
$$

The multifunction $\partial \varphi: X \rightarrow 2^{X^{*}} \backslash\{\emptyset\}$ is known as the generalized (or Clarke) subdifferential of $\varphi$. If $\varphi \in C^{1}(X)$, then $\partial \varphi(x)=\left\{\varphi^{\prime}(x)\right\}$ and if $\varphi$ is convex, then $\partial \varphi(x)$ coincides with the convex subdifferential. The generalized subdifferential has a rich calculus which can be found in [10]. 
A point $x \in X$ is a critical point of the locally Lipschitz function $\varphi$, if $0 \in \partial \varphi(x)$. If $x \in X$ is a critical point, the value $c=\varphi(x)$ is a critical value of $\varphi$. It is easy to check that if $x \in X$ is a local extremum of $\varphi$ (i.e. a local minimum or a local maximum), then $0 \in \partial \varphi(x)$ (i.e. $x \in X$ is a critical point).

We will use the following compactness-type condition:

A locally Lipschitz function $\varphi: X \rightarrow \mathbb{R}$ satisfies the nonsmooth Palais-Smale condition (nonsmooth PS-condition for short) if any sequence $\left\{x_{n}\right\}_{n \geq 1} \subseteq X$ such that $\left\{\varphi\left(x_{n}\right)\right\}_{n \geq 1}$ is bounded and

$$
m_{\varphi}\left(x_{n}\right) \stackrel{d f}{=} \min \left\{\left\|x^{*}\right\|_{X^{*}}: x^{*} \in \partial \varphi\left(x_{n}\right)\right\} \longrightarrow 0 \quad \text { as } n \rightarrow+\infty
$$

has a strongly convergent subsequence.

Since for $\varphi \in C^{1}(X)$ we have $\partial \varphi(x)=\left\{\varphi^{\prime}(x)\right\}$, we see that the above definition is an extension of the smooth PS-condition. It was shown by Cerami [8] and Bartolo, Benci and Fortunato [1] that a slightly more general condition in the smooth setting, suffices to prove the main minimax principles. In the present nonsmooth setting this condition has the following form:

A locally Lipschitz function $\varphi: X \rightarrow \mathbb{R}$ satisfies the nonsmooth Cerami condition (nonsmooth C-condition for short) if any sequence $\left\{x_{n}\right\}_{n \geq 1} \subseteq X$ such that $\left\{\varphi\left(x_{n}\right)\right\}_{n \geq 1}$ is bounded and

$$
\left(1+\left\|x_{n}\right\|_{X}\right) m_{\varphi}\left(x_{n}\right) \longrightarrow 0 \quad \text { as } n \rightarrow+\infty,
$$

has a strongly convergent subsequence.

Recently Kandilakis, Kourogenis and Papageorgiou [21], proved the following multiplicity result extending a corresponding theorem of Brezis and Nirenberg [3].

Theorem 2.1. If $X$ is a reflexive Banach space, $X=Y \oplus V$ with $\operatorname{dim} V<+\infty, \varphi: X \rightarrow \mathbb{R}$ is locally Lipschitz, bounded below, satisfies the nonsmooth C-condition, $\inf _{X} \varphi<0$ and there exists $\rho>0$ such that

$$
\left\{\begin{array}{l}
\varphi(x) \leq 0 \quad \text { if } x \in V,\|x\|_{X} \leq \rho \\
\varphi(x) \geq 0 \quad \text { if } x \in Y,\|x\|_{X} \leq \rho
\end{array},\right.
$$

then $\varphi$ has at least two nontrivial critical points.

Condition 2.1 implies $\varphi(0)=0$. We call condition 2.1 the local linking condition.

Recall that, if $\left\{\lambda_{n}\right\}_{n \geq 1}$ are the distinct eigenvalues of $\left(-\Delta, H_{0}^{1}(Z)\right)$, then $\lambda_{n} \longrightarrow+\infty$ and $\lambda_{1}$ is positive, simple and isolated. Also there is an orthonormal basis $\left\{u_{n}\right\}_{n \geq 1} \subseteq H_{0}^{1}(Z) \cap$ $C^{\infty}(Z)$ of $L^{2}(Z)$, which are eigenfunctions corresponding to the eigenvalues $\left\{\lambda_{n}\right\}_{n \geq 1}$, i.e.

$$
\left\{\begin{array}{l}
-\Delta u_{n}(z)=\lambda_{n} u_{n}(z) \quad \forall z \in Z, \\
\left.u_{n}\right|_{\Gamma}=0
\end{array}\right.
$$

for $n \geq 1$. If the boundary $\Gamma$ of $Z$ is a $C^{k}$-manifold (respectively a $C^{\infty}$-manifold) then $u_{n} \in C^{k}(\bar{Z})$ (respectively $u_{n} \in C^{\infty}(\bar{Z})$ ). The sequence $\left\{\frac{1}{\sqrt{\lambda_{n}}} u_{n}\right\}_{n \geq 1}$ is an orthonormal basis 
of $H_{0}^{1}(Z)$. For every integer $m \geq 1$, let $E\left(\lambda_{m}\right)$ be the eigenspace corresponding to the eigenvalue $\lambda_{m}$. We define

$$
\bar{H}_{m} \stackrel{d f}{=} \bigoplus_{i=1}^{m-1} E\left(\lambda_{i}\right) \quad \text { and } \quad \hat{H}_{m} \stackrel{d f}{=} \overline{\bigoplus_{i=m+1}^{\infty} E\left(\lambda_{i}\right)} .
$$

We have the following orthogonal direct sum decomposition:

$$
H_{0}^{1}(Z)=\bar{H}_{m} \oplus E\left(\lambda_{m}\right) \oplus \hat{H}_{m}
$$

The eigenspace $E\left(\lambda_{m}\right) \subseteq H_{0}^{1}(Z) \cap C^{\infty}(Z)$ has the unique continuation property, namely if $u \in E\left(\lambda_{m}\right)$ is such that $u$ vanishes on a set of positive measure, then $u(z)=0$ for all $z \in Z$.

If we set

$$
V_{m} \stackrel{d f}{=} \bar{H}_{m} \oplus E\left(\lambda_{m}\right) \quad \text { and } \quad W_{m} \stackrel{d f}{=} E\left(\lambda_{m}\right) \oplus \hat{H}_{m},
$$

then on these spaces we have variational characterizations of the eigenvalues (Rayleigh quotients), which can be found in [20].

Let us recall two modes of convergence of sets and functions, which will be used in the proof of our nonsmooth extension of the Castro-Lazer-Thews reduction method. So let $\left(Y_{1}, \tau_{1}\right)$ and $\left(Y_{2}, \tau_{2}\right)$ be two Hausdorff topological spaces ( $\tau_{1}$ and $\tau_{2}$ being the respective topologies). Also let $\left\{G_{n}\right\}_{n \geq 1}$ be a sequence of nonempty subsets of $Y_{1} \times Y_{2}$. We define:

$$
\begin{gathered}
\left(\tau_{1} \times \tau_{2}\right)-\liminf _{n \rightarrow+\infty} G_{n} \stackrel{d f}{=}\left\{(u, v) \in Y_{1} \times Y_{2}: u=\tau_{1}-\lim _{n \rightarrow+\infty} u_{n},\right. \\
\left.v=\tau_{2}-\lim _{n \rightarrow+\infty} v_{n},\left(u_{n}, v_{n}\right) \in G_{n}, n \geq 1\right\}, \\
\left(\tau_{1} \times \tau_{2}\right)-\limsup _{n \rightarrow+\infty} G_{n} \stackrel{d f}{=}\left\{(u, v) \in Y_{1} \times Y_{2}: u=\tau_{1}-\lim _{n \rightarrow+\infty} u_{n_{k}},\right. \\
v=\tau_{2}-\lim _{n \rightarrow+\infty} v_{n_{k}},\left(u_{n_{k}}, v_{n_{k}}\right) \in G_{n_{k}}, \\
\left.n_{i}<n_{i+1} \text { for } i \geq 1\right\} .
\end{gathered}
$$

If

$$
G=\left(\tau_{1} \times \tau_{2}\right)-\liminf _{n \rightarrow+\infty} G_{n}=\left(\tau_{1} \times \tau_{2}\right)-\limsup _{n \rightarrow+\infty} G_{n},
$$

then we say that the sequence $\left\{G_{n}\right\}_{n \geq 1}$ converges in the $\left(\tau_{1} \times \tau_{2}\right)$-sequential Kuratowski sense to $G$ and denote it by

$$
G_{n} \stackrel{K_{\tau_{1}}, \tau_{2}}{\longrightarrow} G \quad \text { as } n \rightarrow+\infty .
$$

Now, let $Y$ be a Banach space and $\left\{\varphi_{n}\right\}_{n \geq 1} \subseteq \overline{\mathbb{R}}^{Y}$ and $\varphi \in \overline{\mathbb{R}}^{Y}$ (with $\overline{\mathbb{R}} \stackrel{d f}{=} \mathbb{R} \cup\{+\infty\}$ ).

We say that the sequence $\{\varphi\}_{n \geq 1}$ converges in the Mosco sense to $\varphi$, denoted by $\varphi_{n} \stackrel{M}{\longrightarrow} \varphi$ if and only if the following two conditions hold: 
(1) for every $y \in Y$ and every sequence $\left\{y_{n}\right\}_{n \geq 1} \subseteq Y$ such that $y_{n} \stackrel{w}{\longrightarrow} y$ in $Y$, we have that

$$
\varphi(y) \leq \liminf _{n \rightarrow+\infty} \varphi_{n}\left(y_{n}\right)
$$

(2) for every $y \in Y$, there exists a sequence $\left\{y_{n}\right\}_{n \geq 1} \subseteq Y$ such that $y_{n} \longrightarrow y$ in $Y$ and $\varphi_{n}\left(y_{n}\right) \longrightarrow \varphi(y)$.

Further analysis of these two notions can be found in [17].

\section{The nonsmooth reduction method}

In this section we extend the Castro, Lazer and Thews reduction method to the present nonsmooth setting. By $2^{*}$ we denote the Sobolev critical exponent defined by

$$
2^{*} \stackrel{d f}{=} \begin{cases}\frac{2 N}{N-2}, & \text { if } \quad N>2 \\ +\infty, & \text { if } \quad N \leq 2 .\end{cases}
$$

Our hypotheses on the nonsmooth potential $j$ are the following:

$\mathrm{H}(\mathrm{j}): j: Z \times \mathbb{R} \longrightarrow \mathbb{R}$ is a function, such that

(i) for all $\zeta \in \mathbb{R}$, the function $Z \ni z \longmapsto j(z, \zeta) \in \mathbb{R}$ is measurable and for almost all $z \in Z$, we have that $j(z, 0)=0$;

(ii) for almost all $z \in Z$, the function $\mathbb{R} \ni \zeta \longmapsto j(z, \zeta) \in \mathbb{R}$ is locally Lipschitz;

(iii) for almost all $z \in Z$, all $\zeta \in \mathbb{R}$ and all $u \in \partial j(z, \zeta)$, we have that $|u| \leq a_{1}(z)+$ $c_{1}|\zeta|^{r-1}$, with $a_{1} \in L^{\infty}(Z), c_{1}>0$ and $1 \leq r<2^{*}$;

(iv) $\lim _{|\zeta| \rightarrow+\infty}[u(\zeta) \zeta-2 j(z, \zeta)]=-\infty$ uniformly for almost all $z \in Z$ and all $u(\zeta) \in$ $\partial j(z, \zeta)$

(v) there exists $l \in L^{\infty}(Z)$, such that for almost all $z \in Z$, we have $l(z) \leq \lambda_{k+1}-\lambda_{k}$ with strict inequality on a set of positive measure and for almost all $z \in Z$, all $\zeta_{1}, \zeta_{2} \in \mathbb{R}$ with $\zeta_{1} \neq \zeta_{2}$ and all $v_{1} \in \partial j\left(z, \zeta_{1}\right), v_{2} \in \partial j\left(z, \zeta_{2}\right)$, we have $\frac{v_{1}-v_{2}}{\zeta_{1}-\zeta_{2}} \leq l(z)$;

(vi) there exist $\beta \in L^{\infty}(Z)_{-}$and $\delta_{0}>0$ such that for some integer $m \in[1, k]$ and for almost all $z \in Z$, we have $\beta(z) \leq \lambda_{m}-\lambda_{k}$, with strict inequality on a set of positive measure and for almost all $z \in Z$ and all $\zeta \in \mathbb{R}$, such that $|\zeta| \leq \delta_{0}$, we have $\lambda_{m-1}-$ $\lambda_{k} \leq \frac{2 j(z, \zeta)}{\zeta^{2}} \leq \beta(z)$

(vii) $0 \leq \liminf _{|\zeta| \rightarrow+\infty} \frac{2 j(z, \zeta)}{\zeta^{2}} \leq \limsup _{|\zeta| \rightarrow+\infty} \frac{2 j(z, \zeta)}{\zeta^{2}} \leq \gamma(z)$ uniformly for almost all $z \in$ $Z$, with $\gamma \in L^{\infty}(Z)_{+}$and $\gamma(z) \leq \lambda_{k+1}-\lambda_{k}$ for almost all $z \in Z$, with strict inequality on a set of positive measure.

Remark 3.1. Hypothesis H(j)(vi) implies that we have double resonance at the origin. The resonance is complete from below and incomplete from above. The same double resonance situation at infinity is implied by hypothesis $\mathrm{H}(\mathrm{j})$ (vii). So hypotheses $\mathrm{H}(\mathrm{j})$ (vi) and $\mathrm{H}(\mathrm{j})$ (vii) together provide the double-double resonance character of our problem. Also note that hypothesis $\mathrm{H}(\mathrm{j})(\mathrm{v})$ permits only downward discontinuities of the derivative of the potential function $j(z, \cdot)$. Recall that for almost all $z \in Z$, the derivative of $j(z, \cdot)$ exists almost everywhere (Rademacher theorem). 
Let $\varphi: H_{0}^{1}(Z) \longrightarrow \mathbb{R}$ be the energy functional defined by

$$
\varphi(x) \stackrel{d f}{=} \frac{1}{2}\|\nabla x\|_{2}^{2}-\frac{\lambda_{k}}{2}\|x\|_{2}^{2}-\int_{Z} j(z, x(z)) \mathrm{d} z \quad \forall x \in H_{0}^{1}(Z)
$$

(by $\|\cdot\|_{p}$ we denote the norm of $L^{p}(Z)$ ). We know that $\varphi$ is locally Lipschitz (see p. 313 of [18]). Since $k \geq 1$ is fixed, for what follows we set

$$
\bar{H} \stackrel{d f}{=} \bar{H}_{k}=\bigoplus_{i=1}^{k-1} E\left(\lambda_{i}\right) \quad \text { and } \quad \hat{H} \stackrel{d f}{=} \hat{H}_{k}=\overline{\bigoplus_{i=k+1}^{\infty} E\left(\lambda_{i}\right)} .
$$

We have that

$$
H_{0}^{1}(Z)=\bar{H} \oplus E\left(\lambda_{k}\right) \oplus \hat{H} .
$$

Also we set

$$
\bar{H}_{0} \stackrel{d f}{=} \bar{H} \oplus E\left(\lambda_{k}\right)=\bigoplus_{i=1}^{k} E\left(\lambda_{i}\right)
$$

and for $u \in \bar{H}_{0}$, we consider the following minimization problem:

$$
\inf _{v \in \hat{H}} \varphi(u+v) .
$$

Since we do not identify $H_{0}^{1}(Z)$ with its dual, we have that

$$
\left(H_{0}^{1}(Z)\right)^{*}=H^{-1}(Z)=\bar{H}_{0}^{*} \oplus \hat{H}^{*} .
$$

We start with a simple lemma which is needed in what follows.

Lemma 3.2. If $n \geq 1$ and $\beta \in L^{\infty}(Z)_{+}$, with

$$
\beta(z) \leq \lambda_{n+1} \text { for a.a. } z \in Z
$$

and the inequality is strict on a set of positive measure, then there exists $\xi_{1}>0$, such that

$$
\|\nabla x\|_{2}^{2}-\int_{Z} \beta(z)|x(z)|^{2} \mathrm{~d} z \geq \xi_{1}\|\nabla x\|_{2}^{2} \quad \forall x \in \hat{H}_{n} .
$$

Proof. Let

$$
\theta(x) \stackrel{d f}{=}\|\nabla x\|_{2}^{2}-\int_{Z} \beta(z)|x(z)|^{2} \mathrm{~d} z \quad \forall x \in \hat{H}_{n} .
$$

By virtue of the variational characterization of the eigenvalues, we have that $\theta \geq 0$. Suppose that the lemma is not true. Because of the positive homogeneity of $\theta$, we can find a sequence $\left\{x_{m}\right\}_{m \geq 1} \subseteq \hat{H}_{n}$, such that $\left\|\nabla x_{m}\right\|_{2}=1$ for $m \geq 1$ and $\theta\left(x_{m}\right) \searrow 0$. By passing to a subsequence if necessary, we may assume that

$$
\begin{array}{ll}
x_{m} \stackrel{w}{\longrightarrow} x_{0} \quad \text { in } H_{0}^{1}(Z), \\
x_{m} \longrightarrow x_{0} \quad \text { in } L^{2}(Z),
\end{array}
$$


with some $x_{0} \in \hat{H}_{n}$. Since the norm in a Banach space is weakly lower semicontinuous, in the limit as $m \rightarrow+\infty$, we obtain

$$
\theta\left(x_{0}\right)=\left\|\nabla x_{0}\right\|_{2}^{2}-\int_{Z} \beta(z)\left|x_{0}(z)\right|^{2} \mathrm{~d} z \leq 0
$$

so

$$
\left\|\nabla x_{0}\right\|_{2}^{2} \leq \int_{Z} \beta(z)\left|x_{0}(z)\right|^{2} \mathrm{~d} z \leq \lambda_{n+1}\left\|x_{0}\right\|_{2}^{2}
$$

and since $x_{0} \in \hat{H}_{n}$, from the variational characterization of $\lambda_{n+1}$, we have that

$$
\left\|\nabla x_{0}\right\|_{2}^{2}=\lambda_{n+1}\left\|x_{0}\right\|_{2}^{2}
$$

If $x_{0}=0$, then taking into account that $\theta\left(x_{m}\right) \rightarrow 0$ we would have that $\left\|\nabla x_{m}\right\|_{2} \rightarrow 0$. Because $\left\|\nabla x_{m}\right\|_{2}=1$ for $m \geq 1$, this is not possible, so $x_{0} \neq 0$. From (3.2), it follows that $x_{0} \in E\left(\lambda_{n+1}\right)$. Then, from the hypothesis that $\beta(z)<\lambda_{n+1}$ on a set of positive measure and from the unique continuity property of $E\left(\lambda_{n+1}\right)$, we have that

$$
\left\|\nabla x_{0}\right\|_{2}^{2} \leq \int_{Z} \beta(z)\left|x_{0}(z)\right|^{2} \mathrm{~d} z<\lambda_{n+1}\left\|x_{0}\right\|_{2}^{2}
$$

a contradiction to 3.2 .

The next proposition essentially extends the Castro-Lazer-Thews reduction method to a nonsmooth setting.

\section{PROPOSITION 3.3.}

If hypotheses $\mathrm{H}(\mathrm{j})$ hold, then there exists a continuous map $\vartheta: \bar{H}_{0} \longrightarrow \hat{H}$, such that for every $u \in \bar{H}_{0}$, we have

$$
\inf _{v \in \hat{H}} \varphi(u+v)=\varphi(u+\vartheta(u))
$$

and $\vartheta(u) \in \hat{H}$ is the unique solution of the operator inclusion

$$
0 \in p_{\hat{H}^{*}} \partial \varphi(u+v)
$$

with $u \in \bar{H}_{0}$ fixed, where $p_{\hat{H}^{*}}$ is the orthogonal projection on $\hat{H}^{*}=\left[\bar{H}_{0}^{*}\right]^{\perp}$.

Proof. For a fixed $u \in \bar{H}_{0}$, let $\varphi_{u}: H_{0}^{1}(Z) \longrightarrow \mathbb{R}$ be defined by

$$
\varphi_{u}(w) \stackrel{d f}{=} \varphi(u+w) \quad \forall w \in H_{0}^{1}(Z) .
$$

For every $w, h \in H_{0}^{1}(Z)$, we have that

$$
\begin{aligned}
\varphi_{u}^{0}(w ; h)=\limsup _{\substack{w^{\prime} \rightarrow w \\
t \searrow 0}} \frac{\varphi_{u}\left(w^{\prime}+t h\right)-\varphi_{u}\left(w^{\prime}\right)}{t} \\
=\limsup _{\substack{w^{\prime} \rightarrow w \\
t \searrow 0}} \frac{\varphi\left(u+w^{\prime}+t h\right)-\varphi\left(u+w^{\prime}\right)}{t}=\varphi^{0}(u+w ; h),
\end{aligned}
$$


so

$$
\partial \varphi_{u}(w)=\partial \varphi(u+w) \quad \forall w \in H_{0}^{1}(Z) .
$$

Let $\hat{i}: \hat{H} \longrightarrow H_{0}^{1}(Z)$ be the inclusion map and let $\hat{\varphi}_{u}: \hat{H} \longrightarrow \mathbb{R}$ be defined by

$$
\hat{\varphi}_{u}(v)=\varphi(u+v) \quad \forall v \in \hat{H} .
$$

We have that $\varphi_{u} \circ \hat{i}=\hat{\varphi}_{u}$ and so

$$
\partial\left(\varphi_{u} \circ \hat{i}\right)(v)=\partial \hat{\varphi}_{u}(v) \quad \forall v \in \hat{H} .
$$

But from the chain rule of Clarke (p. 45-46 of [10]), we have

$$
\partial\left(\varphi_{u} \circ \hat{i}\right)(v) \subseteq p_{\hat{H}^{*}} \partial \varphi_{u}(\hat{i}(v)) \quad \forall v \in \hat{H},
$$

since $\hat{i}^{*}=p_{\hat{H}^{*}}$. Hence, from (3.3) and 3.4, it follows that

$$
\partial \hat{\varphi}_{u}(v) \subseteq p_{\hat{H}^{*}} \partial \varphi_{u}(\hat{i}(v))=p_{\hat{H}^{*}} \partial \varphi(u+v) \quad \forall v \in \hat{H} .
$$

Now we have

$$
x^{*}=A(x)-\lambda_{k} x-h \quad \forall x \in H_{0}^{1}(Z), x^{*} \in \partial \varphi(x),
$$

with $A \in \mathscr{L}\left(H_{0}^{1}(Z), H^{-1}(Z)\right)$ being defined by

$$
\langle A(x), y\rangle_{H_{0}^{1}(Z)} \stackrel{d f}{=} \int_{Z}(\nabla x(z), \nabla y(z))_{\mathbb{R}^{N}} \mathrm{~d} z \quad \forall x, y \in H_{0}^{1}(Z)
$$

and $h \in L^{r^{\prime}}(Z)$ (where $\frac{1}{r}+\frac{1}{r^{\prime}}=1$ ), such that $h(z) \in \partial j(z, x(z))$ for almost all $z \in Z$ (see p. 83 of [10]). So for any $v_{1}, v_{2} \in \hat{H}$ and $x_{1}^{*} \in \partial \hat{\varphi}_{u}\left(v_{1}\right), x_{2}^{*} \in \partial \hat{\varphi}_{u}\left(v_{2}\right)$, we have

$$
x_{i}^{*}=p_{\hat{H}^{*}} A\left(u+v_{i}\right)-\lambda_{k} v_{i}-p_{\hat{H}^{*}} h_{i} \quad \text { for } i \in\{1,2\},
$$

where $h_{i} \in L^{r^{\prime}}(Z) \subseteq H^{-1}(Z)$ (recall $\left.r<2^{*}\right)$ is such that $h_{i}(z) \in \partial j\left(z,\left(u+v_{i}\right)(z)\right)$ for almost all $z \in Z$ and $i \in\{1,2\}$. Since $p_{\hat{H}^{*}}^{*}=\hat{i}$, we have that

$$
\begin{aligned}
& \left\langle p_{\hat{H}^{*}}\left(A\left(u+v_{1}\right)-A\left(u+v_{2}\right)\right), v_{1}-v_{2}\right\rangle_{\hat{H}} \\
& \quad=\left\langle A\left(u+v_{1}\right)-A\left(u+v_{2}\right), \hat{i}\left(v_{1}\right)-\hat{i}\left(v_{2}\right)\right\rangle_{H_{0}^{1}(Z)} \\
& \quad=\left\langle A\left(u+v_{1}\right)-A\left(u+v_{2}\right), v_{1}-v_{2}\right\rangle_{H_{0}^{1}(Z)}=\left\|\nabla v_{1}-\nabla v_{2}\right\|_{2}^{2} .
\end{aligned}
$$

By hypothesis $\mathrm{H}(\mathrm{j})(\mathrm{v})$, we have that

$$
\frac{h_{1}(z)-h_{2}(z)}{v_{1}(z)-v_{2}(z)} \leq l(z) \quad \text { a.e. on }\left\{v_{1} \neq v_{2}\right\} \text {. }
$$

So, from (3.6) and (3.7), it follows that

$$
\begin{aligned}
& \left\langle x_{1}^{*}-x_{2}^{*}, v_{1}-v_{2}\right\rangle_{\hat{H}} \\
& \quad=\left\|\nabla v_{1}-\nabla v_{2}\right\|_{2}^{2}-\lambda_{k}\left\|v_{1}-v_{2}\right\|_{2}^{2}-\int_{Z}\left(h_{1}(z)-h_{2}(z)\right)\left(v_{1}(z)-v_{2}(z)\right) \mathrm{d} z \\
& \quad \geq\left\|\nabla v_{1}-\nabla v_{2}\right\|_{2}^{2}-\lambda_{k}\left\|v_{1}-v_{2}\right\|_{2}^{2}-\int_{Z} l(z)\left|v_{1}(z)-v_{2}(z)\right|^{2} \mathrm{~d} z .
\end{aligned}
$$


By hypothesis $H(j)(v)$, we know that

$$
l(z) \leq \lambda_{k+1}-\lambda_{k} \quad \text { for a.a. } z \in Z,
$$

with strict inequality on a set of positive measure. So we can apply Lemma 3.2 (with $\left.\beta(z)=l(z)+\lambda_{k}\right)$ and obtain $\xi_{1}>0$, such that

$$
\left\langle x_{1}^{*}-x_{2}^{*}, v_{1}-v_{2}\right\rangle_{\hat{H}} \geq \xi_{1}\left\|\nabla v_{1}-\nabla v_{2}\right\|_{2}^{2} .
$$

So the multifunction $v \longmapsto \partial \hat{\varphi}_{u}(v)$ is strongly monotone in the dual pair $\left(\hat{H}, \hat{H}^{*}\right)$. Hence the function $\hat{H} \ni v \longmapsto \hat{\varphi}_{u}(v) \in \mathbb{R}$ is strongly convex, i.e. the function $\hat{H} \ni v \longmapsto \hat{\varphi}_{u}(v)-$ $\frac{\xi_{1}}{2}\|v\|_{H_{0}^{1}(Z)}^{2} \in \mathbb{R}$ is convex (see p. 37 of [10]).

Let $v \in \hat{H}, x^{*} \in \partial \hat{\varphi}_{u}(v)$ and $y^{*} \in \partial \hat{\varphi}_{u}(0)$. From the previous considerations, we have

$$
\begin{aligned}
\left\langle x^{*}, v\right\rangle_{\hat{H}} & =\left\langle x^{*}-y^{*}, v\right\rangle_{\hat{H}}+\left\langle y^{*}, v\right\rangle_{\hat{H}} \\
& \geq \xi_{1}\|\nabla v\|_{2}^{2}-\xi_{2}\left\|y^{*}\right\|_{H^{-1}(Z)}\|\nabla v\|_{2},
\end{aligned}
$$

for some $\xi_{2}>0$, so the multifunction $v \longmapsto \partial \varphi_{u}(v)$ is coercive.

The multifunction $v \longmapsto \partial \hat{\varphi}_{u}(v)$ is maximal monotone (since $\hat{\varphi}_{u}$ is convex). But a maximal monotone, coercive operator is surjective (see p. 322 of [17]). Thus, we can find $v_{0} \in \hat{H}$, such that

$$
0 \in \partial \hat{\varphi}_{u}\left(v_{0}\right) \quad \text { and } \quad \inf _{v \in \hat{H}} \varphi(u+v)=\varphi\left(u+v_{0}\right)
$$

Because of the strong convexity of $\hat{\varphi}_{u}$, we infer that the minimizer $v_{0} \in \hat{H}$ is unique.

Therefore we can define a map $\vartheta: \bar{H}_{0} \longrightarrow \hat{H}$ which to each fixed $u \in \bar{H}_{0}$ assigns the unique solution $v_{0} \in \hat{H}$ of the minimization problem 3.1. Then from 3.5 , we have

$$
0 \in \partial \hat{\varphi}_{u}(\vartheta(u)) \subseteq p_{\hat{H}^{*}} \partial \varphi(u+\vartheta(u))
$$

and

$$
\inf _{v \in \hat{H}} \varphi(u+v)=\varphi(u+\vartheta(u))
$$

Finally, we have to show that $\vartheta$ is continuous. To this end suppose that $u_{n} \rightarrow u$ in $\bar{H}_{0}$. If $v_{n} \longrightarrow v$ in $\hat{H}$, we have $\hat{\varphi}_{u_{n}}\left(v_{n}\right) \longrightarrow \hat{\varphi}_{u}(v)$ (in fact it is easy to see that $\hat{\varphi}_{u_{n}} \longrightarrow \hat{\varphi}_{u}$ in $C(\hat{H})$ ). On the other hand, if $v_{n} \stackrel{w}{\longrightarrow} v$ in $\hat{H}$, by virtue of the weak lower semicontinuity of the norm in a Banach space and the compactness of the embedding $H_{0}^{1}(Z) \subseteq L^{2}(Z)$, we have

$$
\hat{\varphi}_{u}(v) \leq \liminf _{n \rightarrow+\infty} \hat{\varphi}_{u_{n}}\left(v_{n}\right)
$$

(recall the definition of $\varphi$ ). If follows that $\hat{\varphi}_{u_{n}} \stackrel{M}{\longrightarrow} \hat{\varphi}_{u}$ and so by virtue of Theorem 5.6.9 of p. 766 of [17], we have that

$$
\operatorname{Gr} \partial \hat{\varphi}_{u_{n}} \stackrel{K_{s, s}}{\longrightarrow} \operatorname{Gr} \partial \hat{\varphi}_{u} \quad \text { as } n \rightarrow+\infty
$$


Because $0 \in \partial \hat{\varphi}_{u}(\vartheta(u))$, we can find $v_{n}^{*} \in \partial \hat{\varphi}_{v_{n}}\left(v_{n}\right)$, for $n \geq 1$, such that $v_{n} \rightarrow \vartheta(u)$ in $\hat{H}$ and $v_{n}^{*} \longrightarrow 0$ in $\hat{H}^{*}$. Recall that $0 \in \partial \hat{\varphi}_{u_{n}}\left(\vartheta\left(u_{n}\right)\right)$, from the strong monotonicity of $\partial \hat{\varphi}_{u_{n}}$, we have that

$$
\left\langle v_{n}^{*}, v_{n}-\vartheta\left(u_{n}\right)\right\rangle_{\hat{H}} \geq \xi_{1}\left\|v_{n}-\vartheta\left(u_{n}\right)\right\|_{H_{0}^{1}(Z)}^{2}
$$

so

$$
\begin{aligned}
& \left\|\vartheta(u)-\vartheta\left(u_{n}\right)\right\|_{H_{0}^{1}(Z)} \\
& \quad \leq \frac{1}{\xi_{1}}\left\|v_{n}^{*}\right\|_{H^{-1}(Z)}+\left\|v_{n}-\vartheta(u)\right\|_{H_{0}^{1}(Z)} \longrightarrow 0 \quad \text { as } n \rightarrow+\infty
\end{aligned}
$$

and thus we have proved that $\vartheta$ is continuous.

Using Proposition 3.3 we can define the map $\bar{\varphi}: \bar{H}_{0} \longrightarrow \mathbb{R}$, by

$$
\bar{\varphi}(u)=\varphi(u+\vartheta(u)) \quad \forall u \in \bar{H}_{0} .
$$

Note that from the definition of $\vartheta$, for all $u, h \in \bar{H}_{0}$, we have that

$$
\begin{aligned}
\bar{\varphi}(u+h)-\bar{\varphi}(u) & =\varphi(u+h+\vartheta(u+h))-\varphi(u+\vartheta(u)) \\
& \leq \varphi(u+h+\vartheta(u))-\varphi(u+\vartheta(u)) .
\end{aligned}
$$

Similarly from the definition of $\vartheta$, for all $u, h \in \bar{H}_{0}$, we obtain

$$
\begin{aligned}
\bar{\varphi}(u)-\bar{\varphi}(u+h) & =\varphi(u+\vartheta(u))-\varphi(u+h+\vartheta(u+h)) \\
& \leq \varphi(u+\vartheta(u+h))-\varphi(u+h+\vartheta(u+h)) .
\end{aligned}
$$

If follows that $\bar{\varphi}$ is locally Lipschitz (since $\varphi$ is).

Now we will show that

$$
\partial \bar{\varphi}(u) \subseteq p_{\bar{H}_{0}^{*}} \partial \varphi(u+\vartheta(u)) \quad \forall u \in \bar{H}_{0} .
$$

First for all $u, h \in \bar{H}_{0}$, we have

$$
\begin{aligned}
\bar{\varphi}^{0}(u ; h)= & \limsup _{\substack{u^{\prime} \rightarrow u \\
t \searrow 0}} \frac{\bar{\varphi}\left(u^{\prime}+t h\right)-\bar{\varphi}\left(u^{\prime}\right)}{t} \\
= & \limsup _{\substack{u^{\prime} \rightarrow u \\
t \searrow 0}} \frac{\varphi\left(u^{\prime}+t h+\vartheta\left(u^{\prime}+t h\right)\right)-\varphi\left(u^{\prime}+\vartheta\left(u^{\prime}\right)\right)}{t} \\
\leq & \limsup _{\substack{u^{\prime} \rightarrow u \\
t \searrow 0}} \frac{\varphi\left(u^{\prime}+t h+\vartheta\left(u^{\prime}\right)\right)-\varphi\left(u^{\prime}+\vartheta\left(u^{\prime}\right)\right)}{t} \\
\leq & \varphi^{0}(u+\vartheta(u) ; h) .
\end{aligned}
$$


Denoting by $\bar{i}_{0}: \bar{H}_{0} \longrightarrow H_{0}^{1}(Z)$ the inclusion map and noting that $\bar{i}_{0}^{*}=p_{\bar{H}_{0}^{*}}$, for all $u, h \in \bar{H}_{0}$, we have that

$$
\begin{aligned}
& \bar{\varphi}^{0}(u ; h) \leq \varphi^{0}\left(u+\vartheta(u) ; \bar{i}_{0}(h)\right) \\
& \quad=\sup _{u^{*} \in \partial \varphi(u+\vartheta(u))}\left\langle u^{*}, \bar{i}_{0}(h)\right\rangle_{H_{0}^{1}(Z)}=\sup _{u^{*} \in \partial \varphi(u+\vartheta(u))}\left\langle p_{\bar{H}_{0}^{*}}\left(u^{*}\right), h\right\rangle_{\bar{H}_{0}} .
\end{aligned}
$$

Suppose that $u_{0}^{*} \in \partial \bar{\varphi}(u)$. From the definition of the Clarke directional derivative, we have

$$
\left\langle u_{0}^{*}, h\right\rangle_{\bar{H}_{0}} \leq \bar{\varphi}^{0}(u ; h) \quad \forall h \in \bar{H}_{0},
$$

so

$$
\left\langle u_{0}^{*}, h\right\rangle_{\bar{H}_{0}} \leq \sup _{u^{*} \in \partial \varphi(u+\vartheta(u))}\left\langle p_{\bar{H}_{0}^{*}}\left(u^{*}\right), h\right\rangle_{\bar{H}_{0}} \quad \forall h \in \bar{H}_{0}
$$

and thus

$$
u_{0}^{*} \in p_{\bar{H}_{0}^{*}} \partial \varphi(u+\vartheta(u))
$$

Therefore, we obtain [3.9.

Next let $\psi=-\bar{\varphi}$. Then $\psi$ is locally Lipschitz on the finite dimensional space $\bar{H}_{0}$. In the next section working with $\psi$ and using Theorem 2.1] we prove a multiplicity theorem for problem (HVI).

\section{Existence of multiple solutions}

As $m \leq k$ are fixed (see hypothesis $\mathrm{H}(\mathrm{j})(\mathrm{vi})$ ), let us put

$$
Y \stackrel{d f}{=} \bigoplus_{i=1}^{m-1} E\left(\lambda_{i}\right) \quad \text { and } \quad V \stackrel{d f}{=} \bigoplus_{i=m}^{k} E\left(\lambda_{i}\right) .
$$

We have

$$
\bar{H}_{0}=\bar{H} \oplus E\left(\lambda_{k}\right)=Y \oplus V .
$$

The next proposition shows that $\psi=-\bar{\varphi}$ satisfies the local linking condition (see Theorem 2.1.

\section{PROPOSITION 4.1.}

If hypotheses $\mathrm{H}(\mathrm{j})$ hold, then there exists $\delta>0$ such that

$$
\left\{\begin{array}{ll}
\psi(u) \leq 0 & \text { if } u \in V,\|u\|_{H_{0}^{1}(Z)} \leq \delta \\
\psi(u) \geq 0 & \text { if } u \in Y,\|u\|_{H_{0}^{1}(Z)} \leq \delta
\end{array} .\right.
$$

Proof. Because $Y$ is finite dimensional, all norms are equivalent and so we can find $M_{1}>$ 0 , such that

$$
\sup _{z \in Z}|u(z)| \leq M_{1}\|u\|_{H_{0}^{1}(Z)} \quad \forall u \in Y .
$$


Let $\beta \in L^{\infty}(Z)$ and $\delta_{0}>0$ as in hypothesis $\mathrm{H}(\mathrm{j})(\mathrm{vi})$. Thus, if $\delta^{\prime} \stackrel{d f}{=} \frac{\delta_{0}}{M_{1}}$, we have

$$
\sup _{z \in Z}|u(z)| \leq \delta_{0} \quad \forall u \in Y,\|u\|_{H_{0}^{1}(Z)} \leq \delta^{\prime} .
$$

By virtue of hypothesis $\mathrm{H}(\mathrm{j})(\mathrm{vi})$ and the definition of $\vartheta$, for all $u \in Y$ with $\|u\|_{H_{0}^{1}(Z)} \leq \delta^{\prime}$, we have

$$
\begin{aligned}
\psi(u) & =-\bar{\varphi}(u) \geq-\frac{1}{2}\|\nabla u\|_{2}^{2}+\frac{\lambda_{k}}{2}\|u\|_{2}^{2}+\int_{Z} j(z, u(z)) \mathrm{d} z \\
& \geq-\frac{1}{2}\|\nabla u\|_{2}^{2}+\frac{\lambda_{k}}{2}\|u\|_{2}^{2}+\frac{\lambda_{m-1}-\lambda_{k}}{2}\|u\|_{2}^{2} \\
& =-\frac{1}{2}\|\nabla u\|_{2}^{2}+\frac{\lambda_{m-1}}{2}\|u\|_{2}^{2} \geq 0 .
\end{aligned}
$$

Also for all $u \in V$, we have

$$
\begin{aligned}
\psi(u) & =-\varphi(u+\vartheta(u)) \\
& =-\frac{1}{2}\|\nabla(u+\vartheta(u))\|_{2}^{2}+\frac{\lambda_{k}}{2}\|u+\vartheta(u)\|_{2}^{2}+\int_{Z} j(z,(u+\vartheta(u))(z)) \mathrm{d} z .
\end{aligned}
$$

From hypothesis $\mathrm{H}(\mathrm{j})(\mathrm{vi})$, we have that

$$
j(z, \zeta) \leq \frac{1}{2} \beta(z) \zeta^{2} \quad \text { for a.a. } z \in Z \text { and all }|\zeta| \leq \delta_{0},
$$

while by virtue of hypothesis $\mathrm{H}(\mathrm{j})$ (iii) and the Lebourg mean value theorem (see p. 41 of [10] or [24]), we have that

$$
j(z, \zeta) \leq c_{2}|\zeta|^{\eta} \quad \text { for a.a. } z \in Z, \text { all }|\zeta|>\delta_{0},
$$

with some $2<\eta \leq 2^{*}$ and $c_{2}>0$. So finally we can say that

$$
j(z, \zeta) \leq \frac{1}{2} \beta(z) \zeta^{2}+c_{3}|\zeta|^{\eta} \quad \text { for a.a. } z \in Z, \text { all } \zeta \in \mathbb{R}
$$

with $c_{3} \stackrel{d f}{=} c_{2}+\frac{1}{2}\|\beta\|_{\infty}>0$. Using this in 4.1), we obtain

$$
\begin{aligned}
\psi(u) \leq & -\frac{1}{2}\|\nabla(u+\vartheta(u))\|_{2}^{2}+\frac{\lambda_{k}}{2}\|u+\vartheta(u)\|_{2}^{2} \\
& +\frac{1}{2} \int_{Z} \beta(z)|(u+\vartheta(u))(z)|^{2} \mathrm{~d} z+c_{3}\|u+\vartheta(u)\|_{\eta}^{\eta} \quad \forall u \in V .
\end{aligned}
$$

Note that

$$
\lambda_{m-1} \leq \beta(z)+\lambda_{k} \leq \lambda_{m} \quad \text { for almost all } z \in Z,
$$

with the second inequality strict on a set of positive measure. Since $u \in V$ and $\vartheta(u) \in \hat{H}$, $u+\vartheta(u) \in \hat{H}_{m-1}$ and we can apply Lemma 3.2 with $\beta+\lambda_{k}$ to obtain $\xi_{1}>0$ such that

$$
\begin{aligned}
& \|\nabla(u+\vartheta(u))\|_{2}^{2}-\int_{Z}\left(\beta(z)+\lambda_{k}\right)|(u+\vartheta(u))(z)|^{2} \mathrm{~d} z \\
& \quad \geq \xi_{1}\|\nabla(u+\vartheta(u))\|_{2}^{2} \quad \forall v \in V .
\end{aligned}
$$


Using this inequality in 4.2, we have

$$
\psi(u) \leq-\frac{1}{2} \xi_{1}\|\nabla(u+\vartheta(u))\|_{2}^{2}+c_{4}\|\nabla(u+\vartheta(u))\|_{2}^{\eta} \quad \forall v \in V,
$$

for some $c_{4}>0$. Here we have used the Sobolev embedding theorem since $\eta \leq 2^{*}$ and the Poincaré inequality. Because $2<\eta, \vartheta(0)=0$, we can find $\delta^{\prime \prime}>0$, such that

$$
\psi(u) \leq 0 \quad \forall u \in V,\|u\|_{H_{0}^{1}(Z)} \leq \delta^{\prime \prime}
$$

Finally let $\delta \stackrel{d f}{=} \min \left\{\delta^{\prime}, \delta^{\prime \prime}\right\}$ to finish the proof of the proposition.

Since we aim in applying Theorem 2.1 we need to show that $\psi$ satisfies the nonsmooth C-condition. To establish this for $\psi$, we show first that $\varphi$ satisfies the nonsmooth C-condition.

\section{PROPOSITION 4.2.}

If hypotheses $\mathrm{H}(\mathrm{j})$ hold, then $\varphi$ satisfies the nonsmooth $\mathrm{C}$-condition.

Proof. Let $\left\{x_{n}\right\}_{n \geq 1} \subseteq H_{0}^{1}(Z)$ be a sequence, such that

$$
\varphi\left(x_{n}\right) \longrightarrow c \text { and }\left(1+\left\|x_{n}\right\|_{H_{0}^{1}(Z)}\right) m_{\varphi}\left(x_{n}\right) \longrightarrow 0 \text { as } n \rightarrow+\infty \text {. }
$$

Since the norm functional is weakly lower semicontinuous and $\partial \varphi\left(x_{n}\right) \subseteq H^{-1}(Z)$ is weakly compact, from the Weierstrass theorem, we know that there exists $x_{n}^{*} \in \partial \varphi\left(x_{n}\right)$, such that $\left\|x_{n}^{*}\right\|_{H^{-1}(Z)}=m_{\varphi}\left(x_{n}\right)$ for all $n \geq 1$. We know that

$$
x_{n}^{*}=A\left(x_{n}\right)-\lambda_{k} x_{n}-h_{n} \quad \forall n \geq 1,
$$

with $A \in \mathscr{L}\left(H_{0}^{1}(Z), H^{-1}(Z)\right)$ being the maximal monotone operator defined by

$$
\langle A(x), y\rangle_{H_{0}^{1}(Z)} \stackrel{d f}{=} \int_{Z}(\nabla x(z), \nabla y(z))_{\mathbb{R}^{N}} \mathrm{~d} z \quad \forall x, y \in H_{0}^{1}(Z)
$$

and $h_{n} \in L^{r^{\prime}}(Z)$ (with $\frac{1}{r}+\frac{1}{r^{\prime}}=1$ ) is such that $h_{n}(z) \in \partial j\left(z, x_{n}(z)\right)$ for almost all $z \in Z$ (see p. 80 of [10]). From the choice of the sequence $\left\{x_{n}\right\}_{n \geq 1} \subseteq H_{0}^{1}(Z)$, we have

$$
\begin{aligned}
& \left|\left\langle x_{n}^{*}, x_{n}\right\rangle_{H_{0}^{1}(Z)}-2 \varphi\left(x_{n}\right)+2 c\right| \\
& \quad \leq\left\|x_{n}\right\|_{H_{0}^{1}(Z)}\left\|x_{n}^{*}\right\|_{H^{-1}(Z)}+2\left|\varphi\left(x_{n}\right)-c\right| \\
& \quad \leq\left(1+\left\|x_{n}\right\|_{H_{0}^{1}(Z)}\right) m_{\varphi}\left(x_{n}\right)+2\left|\varphi\left(x_{n}\right)-c\right| \longrightarrow 0 \text { as } n \rightarrow+\infty .
\end{aligned}
$$

Note that

$$
\left\langle x_{n}^{*}, x_{n}\right\rangle_{H_{0}^{1}(Z)}=\left\langle A\left(x_{n}\right), x_{n}\right\rangle_{H_{0}^{1}(Z)}-\lambda_{k}\left\|x_{n}\right\|_{2}^{2}-\int_{Z} h_{n}(z) x_{n}(z) \mathrm{d} z
$$

Then using 4.3, we obtain

$$
\begin{aligned}
& \int_{Z}\left(h_{n}(z) x_{n}(z)-2 j\left(z, x_{n}(z)\right)\right) \mathrm{d} z \\
& \quad=2 \varphi\left(x_{n}\right)-\left\langle x_{n}^{*}, x_{n}\right\rangle_{H_{0}^{1}(Z)} \longrightarrow 2 c \quad \text { as } n \rightarrow+\infty .
\end{aligned}
$$


We claim that $\left\{x_{n}\right\}_{n \geq 1} \subseteq H_{0}^{1}(Z)$ is bounded. Suppose that this is not the case. By passing to a subsequence if necessary, we may assume that $\left\|x_{n}\right\|_{H_{0}^{1}(Z)} \longrightarrow+\infty$. Let $y_{n} \stackrel{d f}{=} \frac{x_{n}}{\left\|x_{n}\right\|_{H_{0}^{1}(Z)}}$ for $n \geq 1$. Since $\left\|y_{n}\right\|_{H_{0}^{1}(Z)}=1$, passing to a subsequence if necessary, we may assume that

$$
\begin{aligned}
& y_{n} \stackrel{w}{\longrightarrow} y \quad \text { in } H_{0}^{1}(Z), \\
& y_{n} \longrightarrow y \quad \text { in } L^{2}(Z), \\
& y_{n}(z) \longrightarrow y(z) \quad \text { for a.a. } z \in Z
\end{aligned}
$$

and

$$
\left|y_{n}(z)\right| \leq k(z) \quad \text { for a.a. } z \in Z \text { and all } n \geq 1,
$$

with some $k \in L^{2}(Z)$. Because of hypothesis $\mathrm{H}(\mathrm{j})$ (vii), for a given $\varepsilon>0$, we can find $M_{2}=M_{2}(\varepsilon)>0$ such that for almost all $z \in Z$ and all $|\zeta| \geq M_{2}$, we have

$$
j(z, \zeta) \leq \frac{1}{2}(\gamma(z)+\varepsilon) \zeta^{2} .
$$

Also from hypothesis $\mathrm{H}(\mathrm{j})$ (iii) and the Lebourg mean value theorem, for almost all $z \in Z$ and all $|\zeta|<M_{2}$, we have $|j(z, \zeta)| \leq \xi_{2}$ for some $\xi_{2}>0$. So we can say that for almost all $z \in Z$ and all $\zeta \in \mathbb{R}$, we have

$$
j(z, \zeta) \leq \frac{1}{2}(\gamma(z)+\varepsilon) \zeta^{2}+\xi_{2}
$$

Then for every $n \geq 1$, we have

$$
\begin{aligned}
\frac{\varphi\left(x_{n}\right)}{\left\|x_{n}\right\|_{H_{0}^{1}(Z)}^{2}} & =\frac{1}{2}\left\|\nabla y_{n}\right\|_{2}^{2}-\frac{\lambda_{k}}{2}\left\|y_{n}\right\|_{2}^{2}-\int_{Z} \frac{j\left(z, x_{n}(z)\right)}{\left\|x_{n}\right\|_{H_{0}^{1}(Z)}^{2}} \mathrm{~d} z \\
& \geq \frac{c_{4}}{2}-\frac{\lambda_{k}}{2}\left\|y_{n}\right\|_{2}^{2}-\frac{1}{2} \int_{Z} \gamma(z) y_{n}(z)^{2} \mathrm{~d} z-\frac{\varepsilon}{2}\left\|y_{n}\right\|_{2}^{2}-\frac{\xi_{2}|Z|}{\left\|x_{n}\right\|_{H_{0}^{1}(Z)}^{2}},
\end{aligned}
$$

for some $c_{4}>0$ (by the Poincaré inequality). Passing to the limit as $n \rightarrow+\infty$, we obtain

$$
0 \geq \frac{1}{2}\left(c_{4}-\left(\lambda_{k}+\|\gamma\|_{\infty}+\varepsilon\right)\|y\|_{2}^{2}\right)
$$

and thus $y \neq 0$.

Because of hypothesis $\mathrm{H}(\mathrm{j})(\mathrm{iv})$, we can find $M_{3}>0$ such that for almost all $z \in Z$, all $|\zeta| \geq M_{3}$ and all $u \in \partial j(z, \zeta)$, we have

$$
u \zeta-2 j(z, \zeta) \leq-1 .
$$

On the other hand, as above, for almost all $z \in Z$ and all $|\zeta|<M_{3}$, we have $|j(z, \zeta)| \leq \xi_{3}$ for some $\xi_{3}>0$. Thus using hypothesis $\mathrm{H}(\mathrm{j})(\mathrm{iii})$, we see that for almost all $z \in Z$, all $|\zeta|<M_{3}$ and all $u \in \partial j(z, \zeta)$, we have

$$
|u \zeta-2 j(z, \zeta)| \leq \xi_{4},
$$


for some $\xi_{4}>0$. Thus finally, for almost all $z \in Z$, all $\zeta \in \mathbb{R}$ and all $u \in \partial j(z, \zeta)$, we have

$$
u \zeta-2 j(z, \zeta) \leq \xi_{4}
$$

Let $C \stackrel{d f}{=}\{z \in Z: y(z) \neq 0\}$. Evidently $|C|_{N}>0$ (with $|\cdot|_{N}$ being the Lebesgue measure on $\mathbb{R}^{N}$ ) and for all $z \in C$, we have that

$$
\left|x_{n}(z)\right| \longrightarrow+\infty \quad \text { as } n \rightarrow+\infty .
$$

From (4.6) and by virtue of Lemma 1 of [35], for a given $\delta \in\left(0,|C|_{N}\right)$, we can find a measurable subset $C_{1} \subseteq C$, such that $\left|C \backslash C_{1}\right|_{N}<\delta$ and $\left|x_{n}(z)\right| \longrightarrow+\infty$ uniformly for all $z \in C_{1}$. Using hypothesis $\mathrm{H}(\mathrm{j})(\mathrm{iv})$, we infer that

$$
\int_{C_{1}}\left(h_{n}(z) x_{n}(z)-2 j\left(z, x_{n}(z)\right)\right) \mathrm{d} z \longrightarrow-\infty \quad \text { as } n \rightarrow+\infty .
$$

From (4.5), we have

$$
h_{n}(z) x_{n}(z)-2 j\left(z, x_{n}(z)\right) \leq \xi_{4} \quad \text { for a.a. } z \in Z \backslash C_{1},
$$

and so

$$
\begin{aligned}
& \int_{Z}\left(h_{n}(z) x_{n}(z)-2 j\left(z, x_{n}(z)\right)\right) \mathrm{d} z \\
& \quad=\int_{C_{1}}\left(h_{n}(z) x_{n}(z)-2 j\left(z, x_{n}(z)\right)\right) \mathrm{d} z+\xi_{4}\left|\left(Z \backslash C_{1}\right)_{c}\right|_{N} \longrightarrow-\infty,
\end{aligned}
$$

as $n \rightarrow+\infty$, which contradicts (4.4). This proves the boundedness of $\left\{x_{n}\right\} \subseteq H_{0}^{1}(Z)$. So, passing to a subsequence if necessary, we may assume that

$$
\begin{array}{ll}
x_{n} \stackrel{w}{\longrightarrow} x_{0} & \text { in } H_{0}^{1}(Z) \\
x_{n} \longrightarrow x_{0} & \text { in } L^{2}(Z),
\end{array}
$$

for some $x_{0} \in H_{0}^{1}(Z)$. From the choice of the sequence $\left\{x_{n}\right\}_{n \geq 1} \subseteq H_{0}^{1}(Z)$, we have

$$
\begin{aligned}
\left|\left\langle x_{n}^{*}, x_{n}-x_{0}\right\rangle_{H_{0}^{1}(Z)}\right| & \\
= & \mid\left\langle A\left(x_{n}\right), x_{n}-x_{0}\right\rangle_{H_{0}^{1}(Z)}-\lambda_{k}\left\langle x_{n}^{*}, x_{n}-x\right\rangle_{H_{0}^{1}(Z)} \\
& \quad-\int_{Z} h_{n}(z)\left(x_{n}(z)-x_{0}(z)\right) \mathrm{d} z \mid \\
\leq & m_{\varphi}\left(x_{n}\right)\left\|x_{n}-x_{0}\right\|_{H_{0}^{1}(Z)} \longrightarrow 0 \quad \text { as } n \rightarrow+\infty .
\end{aligned}
$$

But

$$
\lambda_{k}\left\langle x_{n}^{*}, x_{n}-x\right\rangle_{H_{0}^{1}(Z)}+\int_{Z} h_{n}(z)\left(x_{n}(z)-x_{0}(z)\right) \mathrm{d} z \longrightarrow 0 \quad \text { as } n \rightarrow+\infty .
$$

So it follows that

$$
\lim _{n \rightarrow+\infty}\left\langle A\left(x_{n}\right), x_{n}-x_{0}\right\rangle_{H_{0}^{1}(Z)}=0 .
$$


From the maximal monotonicity of $A$, we have

$$
\left.\left\langle A\left(x_{n}\right), x_{n}\right\rangle_{H_{0}^{1}(Z)} \longrightarrow\left\langle A\left(x_{0}\right), x_{0}\right)\right\rangle_{H_{0}^{1}(Z)}
$$

and so

$$
\left\|\nabla x_{n}\right\|_{2} \longrightarrow\left\|\nabla x_{0}\right\|_{2}
$$

Because $\nabla x_{n} \stackrel{w}{\longrightarrow} \nabla x_{0}$ in $L^{2}\left(Z ; \mathbb{R}^{N}\right)$, from the Kadec-Klee property of Hilbert spaces, we conclude that $\nabla x_{n} \longrightarrow \nabla x_{0}$ in $L^{2}\left(Z ; \mathbb{R}^{N}\right)$, hence $x_{n} \longrightarrow x_{0}$ in $H_{0}^{1}(Z)$ as $n \rightarrow+\infty$.

Using this proposition, we can establish that $\bar{\varphi}$ satisfies the nonsmooth C-condition.

\section{PROPOSITION 4.3.}

If hypotheses $\mathrm{H}(\mathrm{j})$ hold, then $\bar{\varphi}$ satisfies the nonsmooth $\mathrm{C}$-condition.

Proof. Let $c \in \mathbb{R}$ and let $\left\{u_{n}\right\}_{n \geq 1} \subseteq \bar{H}_{0}$ be a sequence, such that

$$
\bar{\varphi}\left(u_{n}\right) \longrightarrow c \quad \text { and } \quad\left(1+\left\|u_{n}\right\|_{H_{0}^{1}(Z)}\right) m_{\bar{\varphi}}\left(u_{n}\right) \longrightarrow 0 \quad \text { as } n \rightarrow+\infty .
$$

As before we can find $\bar{v}_{n}^{*} \in \partial \bar{\varphi}\left(u_{n}\right)$, such that $m_{\bar{\varphi}}\left(u_{n}\right)=\left\|\bar{v}_{n}^{*}\right\|_{H^{-1}(Z)}$. By virtue of [3.9], we can find $v_{n}^{*} \in \partial \varphi\left(u_{n}+\vartheta\left(u_{n}\right)\right)$, such that

$$
\bar{v}_{n}^{*}=p_{\bar{H}_{0}^{*}} v_{n}^{*} \quad \forall n \geq 1 .
$$

Recall that, by Proposition $3.30 \in p_{\hat{H}^{*}} \partial \varphi\left(u_{n}+\vartheta\left(u_{n}\right)\right)$, for $n \geq 1$. Then using hypothesis $\mathrm{H}(\mathrm{j})(\mathrm{v})$ we have $m_{\varphi}\left(u_{n}+\vartheta\left(u_{n}\right)\right) \leq m_{\bar{\varphi}}\left(u_{n}\right)$. Therefore,

$$
\left(1+\left\|u_{n}\right\|_{H_{0}^{1}(Z)}\right)\left\|v_{n}^{*}\right\|_{H_{0}^{1}(Z)} \longrightarrow 0 \quad \text { with } v_{n}^{*} \in \partial \varphi\left(u_{n}+\vartheta\left(u_{n}\right)\right) .
$$

But from Proposition 4.2 we know that $\varphi$ satisfies the nonsmooth C-condition. So we can extract a subsequence of $\left\{u_{n}\right\}_{n \geq 1}$, which is strongly convergent. This proves the proposition.

\section{PROPOSITION 4.4.}

If hypotheses $\mathrm{H}(\mathrm{j})$ hold, then $\psi$ is bounded below.

Proof. We show that $-\left(\left.\varphi\right|_{\bar{H}_{0}}\right)$ is bounded below. Then because $-\psi=\bar{\varphi} \leq\left(\left.\varphi\right|_{\bar{H}_{0}}\right)$, we can conclude that $\psi$ is bounded below. To this end we proceed by contradiction. Suppose that $-\left(\left.\varphi\right|_{\bar{H}_{0}}\right)$ is not bounded below. Then we can find $x_{n} \in \bar{H}_{0}$, such that

$$
\varphi\left(x_{n}\right) \geq n \quad \forall n \geq 1
$$

and

$$
\left\|x_{n}\right\|_{H_{0}^{1}(Z)} \longrightarrow+\infty .
$$

By virtue of hypothesis $\mathrm{H}(\mathrm{j})$ (vii), for a given $\varepsilon>0$, we can find $M_{4}=M_{4}(\varepsilon)>0$, such that for almost all $z \in Z$ and all $|\zeta| \geq M_{4}$, we have

$$
-\frac{\varepsilon}{2} \zeta^{2} \leq j(x, \zeta)
$$


On the other hand, as before via Lebourg mean value theorem, we can find $\xi_{5}>0$, such that for almost all $z \in Z$ and all $|\zeta| \leq M_{4}$, we have

$$
|j(z, \zeta)| \leq \xi_{5} .
$$

So finally we see that

$$
-\frac{\varepsilon}{2} \zeta^{2}-\xi_{5} \leq j(z, \zeta) \quad \text { for a.a. } z \in Z, \text { all } \zeta \in \mathbb{R}
$$

Let

$$
x_{n}=\bar{x}_{n}+\overline{\bar{x}}_{n}, \quad \text { with } \bar{x}_{n} \in \bar{H}, \overline{\bar{x}}_{n} \in E\left(\lambda_{k}\right), \quad n \geq 1 .
$$

First assume that

$$
\frac{\left\|\nabla \bar{x}_{n}\right\|_{2}}{\left\|\nabla x_{n}\right\|_{2}} \longrightarrow \mu \neq 0 \quad \text { as } n \rightarrow+\infty .
$$

Exploiting the orthogonality relations, the fact that $\left\|\nabla \overline{\bar{x}}_{n}\right\|_{2}^{2}=\lambda_{k}\left\|\overline{\bar{x}}_{n}\right\|_{2}^{2}$ and estimate [4.8), we have

$$
\begin{aligned}
\varphi\left(x_{n}\right) & =\frac{1}{2}\left\|\nabla \bar{x}_{n}\right\|_{2}^{2}-\frac{\lambda_{k}}{2}\left\|\bar{x}_{n}\right\|_{2}^{2}-\int_{Z} j\left(z, x_{n}(z)\right) \mathrm{d} z \\
& \leq \frac{1}{2}\left\|\nabla \bar{x}_{n}\right\|_{2}^{2}-\frac{\lambda_{k}}{2}\left\|\bar{x}_{n}\right\|_{2}^{2}+\frac{\varepsilon}{2}\left\|x_{n}\right\|_{2}^{2}+\xi_{5}|Z|_{N} .
\end{aligned}
$$

Thus from the variational characterization of the eigenvalues we get

$$
\begin{aligned}
\varphi\left(x_{n}\right) & \leq \frac{1}{2}\left(1-\frac{\lambda_{k}}{\lambda_{k-1}}\right)\left\|\nabla \bar{x}_{n}\right\|_{2}^{2}+\frac{\varepsilon}{2}\left\|x_{n}\right\|_{2}^{2}+\xi_{5}|Z|_{N} \\
& \leq \frac{1}{2}\left\|\nabla x_{n}\right\|_{2}^{2}\left(\left(1-\frac{\lambda_{k}}{\lambda_{k-1}}\right) \frac{\left\|\nabla \bar{x}_{n}\right\|_{2}^{2}}{\left\|\nabla x_{n}\right\|_{2}^{2}}+\frac{\varepsilon}{\lambda_{k}}\right)+\xi_{5}|Z|_{N} .
\end{aligned}
$$

Since by hypothesis, we have that $\left\|\nabla x_{n}\right\|_{2} \longrightarrow+\infty$, so from (4.9) and recalling that $\lambda_{k-1}<\lambda_{k}$, by passing to the limit as $n \rightarrow+\infty$ in 4.10, we see that $\varphi\left(x_{n}\right) \longrightarrow-\infty$ as $n \rightarrow+\infty$, a contradiction to 4.7.

Next assume that

$$
\frac{\left\|\nabla \bar{x}_{n}\right\|_{2}}{\left\|\nabla x_{n}\right\|_{2}} \longrightarrow 0 \quad \text { as } n \rightarrow+\infty .
$$

By virtue of hypothesis $\mathrm{H}(\mathrm{j})(\mathrm{iv})$, for a given $\eta>0$, we can find $M_{5}=M_{5}(\eta)>0$, such that for almost all $z \in Z$, all $|\zeta| \geq M_{5}$ and all $u \in \partial j(z, \zeta)$, we have

$$
u \zeta-2 j(z, \zeta) \leq-\eta \text {. }
$$

From p. 48 of [10], we know that for almost all $z \in Z$ and all $\zeta>0$, the function $\zeta \longmapsto \frac{j(z, \zeta)}{\zeta^{2}}$ is locally Lipschitz and we have that

$$
\begin{aligned}
\partial\left(\frac{j(z, \zeta)}{\zeta^{2}}\right) & =\frac{\zeta^{2} \partial j(z, \zeta)-2 \zeta j(z, \zeta)}{\zeta^{4}} \\
& =\frac{\zeta \partial j(z, \zeta)-2 j(z, \zeta)}{\zeta^{3}} .
\end{aligned}
$$


Using (4.12), we see that for almost all $z \in Z$, all $\zeta \geq M_{5}$ and all $v \in \partial\left(\frac{j(z, \zeta)}{\zeta^{2}}\right)$, we have

$$
v \leq-\frac{\eta}{\zeta^{3}}
$$

Since for all $z \in Z \backslash E$, with $|E|_{N}=0$, the function $\zeta \longmapsto \frac{j(z, \zeta)}{\zeta^{2}}$ is locally Lipschitz on $\left[M_{5},+\infty\right)$, it is differentiable at every $x \in\left[M_{5},+\infty\right) \backslash L(z)$ with some $|L(z)|_{1}=0$. We set

$$
\xi_{0}(z, \zeta) \stackrel{d f}{=} \begin{cases}\frac{\mathrm{d}}{\mathrm{d} z}\left(\frac{j(z, \zeta)}{\zeta^{2}}\right), & \text { if } \quad x \in\left[M_{5},+\infty\right) \backslash L(z), \\ 0, & \text { if } \quad x \in L(z) .\end{cases}
$$

For all $z \in Z \backslash E$ and all $\zeta \in\left[M_{5},+\infty\right) \backslash L(z)$, we have $\xi_{0}(z, \zeta) \in \partial\left(\frac{j(z, \zeta)}{\zeta^{2}}\right)$ and so

$$
\xi_{0}(z, \zeta) \leq-\frac{\eta}{\zeta^{3}}
$$

Let $\tau, \bar{\tau} \in\left[M_{5},+\infty\right)$, with $\tau \leq \bar{\tau}$. Integrating the above inequality with respect to $\zeta \in \mathbb{R}$ on the interval $[\tau, \bar{\tau}]$, we obtain

$$
\frac{j(z, \bar{\tau})}{\bar{\tau}^{2}}-\frac{j(z, \tau)}{\tau^{2}} \leq-\frac{\eta}{2}\left(\frac{1}{\tau^{2}}-\frac{1}{\bar{\tau}^{2}}\right) .
$$

Let $\bar{\tau} \longrightarrow+\infty$ and using hypothesis $\mathrm{H}(\mathrm{j})(\mathrm{vii})$, we obtain

$$
\frac{j(z, \tau)}{\tau^{2}} \geq \frac{\eta}{2 \tau^{2}}
$$

and thus, for almost all $z \in Z$ and all $\tau \geq M_{5}$, we have that

$$
j(z, \tau) \geq \frac{\eta}{2} .
$$

Since $\eta>0$ was arbitrary, it follows that $j(z, \tau) \longrightarrow+\infty$ as $\tau \rightarrow+\infty$ uniformly for almost all $z \in Z$. Similarly we show that $j(z, \tau) \longrightarrow+\infty, \tau \rightarrow-\infty$ uniformly for almost all $z \in Z$. Therefore

$$
j(z, \zeta) \longrightarrow+\infty \text { as }|\zeta| \rightarrow+\infty \text { uniformly for a.a. } z \in Z .
$$

From [1] (see the proof of Lemma 3.2) or [33], we have that for a given $\delta>0$ we can find $\xi_{\delta}>0$, such that

$$
\left|\left\{z \in Z:|v(z)|<\xi_{\delta}\|v\|_{H_{0}^{1}(Z)}\right\}\right|_{N}<\delta \quad \forall v \in E\left(\lambda_{k}\right) .
$$

Let us set

$$
C_{n} \stackrel{d f}{=}\left\{z \in Z:\left|\overline{\bar{x}}_{n}(z)\right| \geq \xi_{\delta}\left\|\overline{\bar{x}}_{n}\right\|_{H_{0}^{1}(Z)}\right\} \quad \forall n \geq 1 .
$$

We have that $\left|Z \backslash C_{n}\right|_{N}<\delta$. 
Let us establish that the sequence $\left\{\bar{x}_{n}\right\}_{n \geq 1}$ is bounded in $H_{0}^{1}(Z)$. Arguing by contradiction, assume that along a relabeled subsequence we have $\left\|\bar{x}_{n}\right\|_{H_{0}^{1}(Z)} \rightarrow+\infty$. Recall that

$$
\varphi\left(x_{n}\right) \leq \frac{1}{2}\left(1-\frac{\lambda_{k}}{\lambda_{k-1}}\right)\left\|\nabla \bar{x}_{n}\right\|_{2}^{2}-\int_{Z} j\left(z, x_{n}(z)\right) \mathrm{d} z \quad \forall n \geq 1 .
$$

Using (4.13) we find a constant $\alpha>0$ such that $j(z, \zeta) \geq 0$ a.a. $z \in Z, \forall|\zeta|>\alpha$. We see that

$$
\begin{aligned}
-\int_{Z} j\left(z, x_{n}(z)\right) \mathrm{d} z= & -\int_{\left\{z \in Z:\left|x_{n}(z)\right|>\alpha\right\}} j\left(z, x_{n}(z)\right) \mathrm{d} z \\
& -\int_{\left\{z \in Z:\left|x_{n}(z)\right| \leq \alpha\right\}} j\left(z, x_{n}(z)\right) \mathrm{d} z \\
\leq & -\int_{\left\{z \in Z:\left|x_{n}(z)\right| \leq \alpha\right\}} j\left(z, x_{n}(z)\right) \mathrm{d} z \\
\leq & k_{0}|Z|_{N},
\end{aligned}
$$

where $k_{0}>0$ is a constant. In writing the last inequality above we made use of assumption $\mathrm{H}(\mathrm{j})$ (iii) and Lebourg's mean value theorem. Then we obtain the estimate

$$
\varphi\left(x_{n}\right) \leq \frac{1}{2}\left(1-\frac{\lambda_{k}}{\lambda_{k-1}}\right)\left\|\nabla \bar{x}_{n}\right\|_{2}^{2}+k_{0}|Z|_{N} .
$$

Since $\lambda_{k-1}<\lambda_{k}$ and we supposed that $\left\|\bar{x}_{n}\right\|_{H_{0}^{1}(Z)} \rightarrow+\infty$, we arrive at the conclusion that $\varphi\left(x_{n}\right) \rightarrow-\infty$ as $n \rightarrow+\infty$. This contradicts relation 4.7), and thus the sequence $\left\{\bar{x}_{n}\right\}_{n \geq 1}$ is bounded in $H_{0}^{1}(Z)$.

Because $\bar{x}_{n} \in \bar{H}$ and the space $\bar{H}$ is finite dimensional, it follows from the boundedness of the sequence $\left\{\bar{x}_{n}\right\}_{n \geq 1} \subseteq H_{0}^{1}(Z)$ that we can find $c_{5}>0$ such that

$$
\left|\bar{x}_{n}(z)\right| \leq c_{5} \quad \forall z \in Z, n \geq 1 .
$$

From (4.13), we know that for a given $\eta_{1}>0$ we can find $M_{6}=M_{6}\left(\eta_{1}\right)>0$ such that

$$
j(z, \zeta) \geq \eta_{1} \quad \text { for a.a. } z \in Z, \text { all }|\zeta| \geq M_{6} .
$$

Let

$$
D_{n} \stackrel{d f}{=}\left\{z \in Z:\left|x_{n}(z)\right| \geq M_{6}\right\} \quad \forall n \geq 1 .
$$

If $z_{0} \in C_{n}$, then

$$
\left|x_{n}\left(z_{0}\right)\right| \geq\left|\overline{\bar{x}}_{n}\left(z_{0}\right)\right|-\left|\bar{x}_{n}\left(z_{0}\right)\right| \geq \xi_{\delta}\left\|\overline{\bar{x}}_{n}\right\|_{H_{0}^{1}(Z)}-c_{5} .
$$

Because of 4.11), we must have that $\left\|\overline{\bar{x}}_{n}\right\|_{H_{0}^{1}(Z)} \longrightarrow+\infty$ as $n \rightarrow+\infty$. So there exists $n_{0} \geq 1$ large enough such that

$$
\xi_{\delta}\left\|\overline{\bar{x}}_{n}\right\|_{H_{0}^{1}(Z)}-c_{5} \geq M_{6} \quad \forall n \geq n_{0}
$$

and so $z_{0} \in D_{n}$ for $n \geq n_{0}$, i.e.

$$
C_{n} \subseteq D_{n} \quad \forall n \geq n_{0} .
$$


Then using (4.8) and the fact that $\left|Z \backslash D_{n}\right|<\delta$ (as $Z \backslash D_{n} \subseteq Z \backslash C_{n}$ ), for $n \geq n_{0}$, we have that

$$
\begin{aligned}
\int_{Z} j\left(z, x_{n}(z)\right) \mathrm{d} z & =\int_{D_{n}} j\left(z, x_{n}(z)\right) \mathrm{d} z+\int_{Z \backslash D_{n}} j\left(z, x_{n}(z)\right) \mathrm{d} z \\
& \geq \eta_{1}\left|D_{n}\right|_{N}-\left(\frac{\varepsilon}{2} M_{6}^{2}+\xi_{5}\right)\left|Z \backslash D_{n}\right|_{N} \\
& \geq \eta_{1}\left|D_{n}\right|_{N}-\left(\frac{\varepsilon}{2} M_{6}^{2}+\xi_{5}\right) \delta \\
& \geq \eta_{1}\left(|Z|_{N}-\delta\right)-\left(\frac{\varepsilon}{2} M_{6}^{2}+\xi_{5}\right) \delta
\end{aligned}
$$

so

$$
\liminf _{n \rightarrow+\infty} \int_{Z} j\left(z, x_{n}(z)\right) \mathrm{d} z \geq \eta_{1}\left(|Z|_{N}-\delta\right)-\left(\frac{\varepsilon}{2} M_{6}^{2}+\xi_{5}\right) \delta .
$$

Since $\delta>0$ is arbitrary, we let $\delta \searrow 0$. We obtain

$$
\liminf _{n \rightarrow+\infty} \int_{Z} j\left(z, x_{n}(z)\right) \mathrm{d} z \geq \eta_{1}|Z|_{N} .
$$

Because $\eta_{1}>0$ is arbitrary, we conclude that

$$
\int_{Z} j\left(z, x_{n}(z)\right) \mathrm{d} z \longrightarrow+\infty \quad \text { as } n \rightarrow+\infty .
$$

From the choice of the sequence $\left\{x_{n}\right\}_{n \geq 1} \subseteq \bar{H}_{0}$, we have

$$
n \leq \varphi\left(x_{n}\right) \leq \frac{1}{2}\left(1-\frac{\lambda_{k}}{\lambda_{k-1}}\right)\left\|\nabla \bar{x}_{n}\right\|_{2}^{2}-\int_{Z} j\left(z, x_{n}(z)\right) \mathrm{d} z \longrightarrow-\infty,
$$

a contradiction. Therefore $-\left(\left.\varphi\right|_{\bar{H}_{0}}\right)$ is bounded below and so $\psi$ is bounded below too.

Now we are ready for our multiplicity result.

Theorem 4.5. If hypotheses $\mathrm{H}(\mathrm{j})$ hold, then problem (HVI) has at least two nontrivial solutions.

Proof. If $\inf _{\bar{H}_{0}} \psi=0($ remark that $\psi(0)=0)$, then by virtue of Proposition 4.1 all $x \in V$ with $\|x\|_{V} \leq \delta$ are critical points of $\psi$.

So assume that $\inf _{\bar{H}_{0}} \psi<0$. By virtue of Propositions 4.1 4.3 and 4.4 and since $\psi(0)=$ 0 , we can apply Theorem 2.1 and obtain two nontrivial critical points $x_{1}, x_{2} \in \bar{H}_{0}$ of $\psi$, i.e.

$$
0 \in \partial \psi\left(x_{i}\right) \quad \text { for } i=1,2 .
$$

Hence $0 \in \partial \bar{\varphi}\left(x_{i}\right)$ for $i=1,2$ and finally from 3.9), we have

$$
0 \in p_{\bar{H}_{0}^{*}} \partial \varphi\left(x_{i}+\vartheta\left(x_{i}\right)\right) \quad \text { for } i=1,2
$$

(see the definition of $\vartheta$ in Proposition 3.3. Recall that $0 \in p_{\hat{H}^{*}} \partial \varphi\left(x_{i}+\vartheta\left(x_{i}\right)\right)$ for $i=1,2$, where $\hat{H}=\bar{H}_{0}^{\perp}$. Therefore we have $0 \in \partial \varphi\left(x_{i}+\vartheta\left(x_{i}\right)\right)$ for $i=1,2$. Set $u_{i}=x_{i}+\vartheta\left(x_{i}\right)$ for $i=1,2$. Then $u_{1}$ and $u_{2}$ are two nontrivial critical points of $\varphi$, and thus they are two nontrivial solutions of (HVI). 


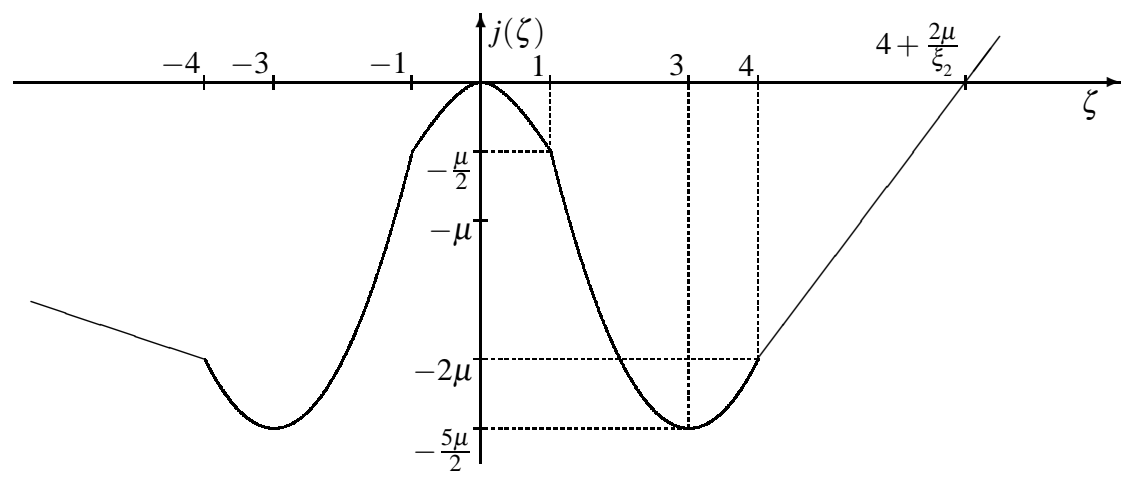

Figure 1. Potential function.

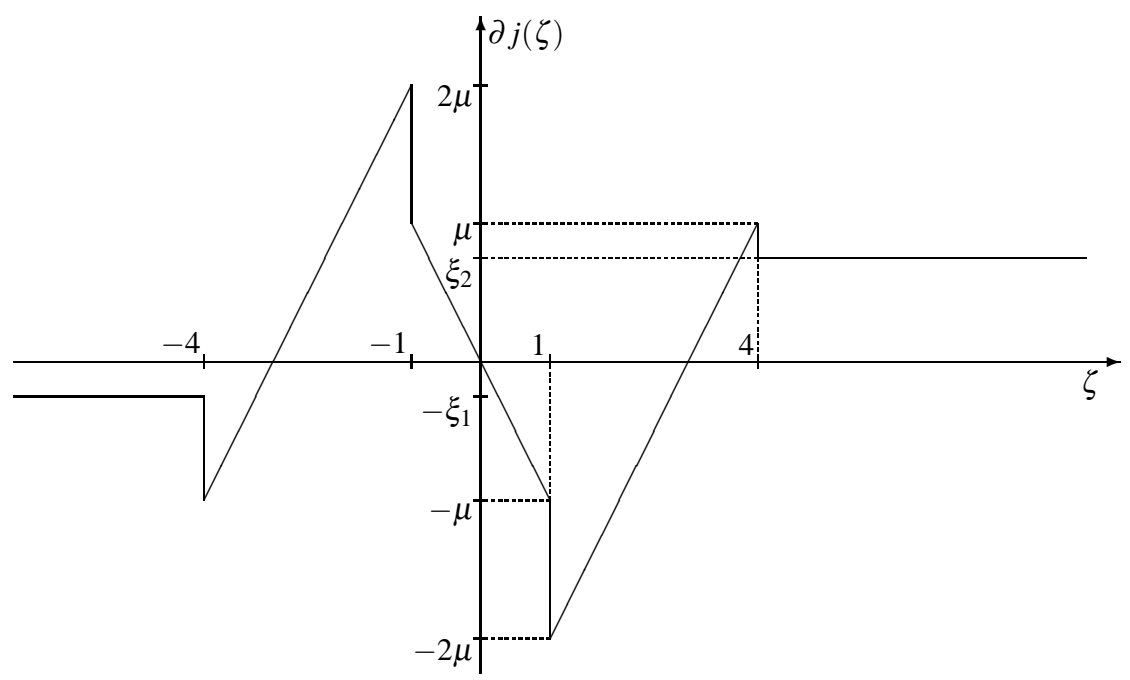

Figure 2. Subdifferential of the potential.

Remark 4.6. An example of a nonsmooth locally Lipschitz potential satisfying hypotheses $\mathrm{H}(\mathrm{j})$ is the following. For simplicity we drop the $z$-dependence (see figure 1 for $j$ and figure 2 for the Clarke subdifferential $\partial j$ ).

$$
j(\zeta) \stackrel{d f}{=} \begin{cases}-\xi_{1} \zeta-2 \mu-4 \xi_{1}, & \text { if } \quad \zeta<-4 \\ \frac{\mu}{2} \zeta^{2}+3 \mu \zeta+2 \mu, & \text { if } \quad-4 \leq \zeta<-1 \\ -\frac{\mu}{2} \zeta^{2}, & \text { if } \quad-1 \leq \zeta<1 \\ \frac{\mu}{2} \zeta^{2}-3 \mu \zeta+2 \mu, & \text { if } \quad 1 \leq \zeta<4 \\ \xi_{2} \zeta-2 \mu-4 \xi_{2}, & \text { if } \quad 4 \leq \zeta .\end{cases}
$$


Here $\lambda_{k}-\lambda_{m}<\mu<\min \left\{\lambda_{k}-\lambda_{m-1}, \lambda_{k+1}-\lambda_{k}\right\}, 0<\xi_{1}, \xi_{2}<\mu$. All the assumptions (i)-(vii) in $\mathrm{H}(\mathrm{j})$ are verified. For instance, assumption $\mathrm{H}(\mathrm{j})(\mathrm{v})$ holds with $l(z) \equiv \mu$. In this case we have resonance at $\pm \infty$ since $\frac{j(\zeta)}{\zeta^{2}} \longrightarrow 0$ as $|\zeta| \rightarrow+\infty$. Another possibility is the function $j(x)=\max \left\{\frac{\xi}{2} x^{2}+c|x|, \frac{\xi}{2}|x|\right\}$ with $\xi<\lambda_{k+1}-\lambda_{k}, c \leq \frac{\xi}{2}$.

\section{Acknowledgement}

The authors wish to thank the referee for his constructive remarks. The first author (LG) is an award holder of the NATO Science Fellowship Programme, which was spent in the National Technical University of Athens.

\section{References}

[1] Bartolo P, Benci V and Fortunato D, Abstract critical point theorems and applications to some nonlinear problems with 'strong' resonance at infinity, Nonlin. Anal. 7 (1983) 981-1012

[2] Berestycki H and de Figueiredo D, Double resonance in semilinear elliptic problems, Comm. Partial Diff. Eqns 6 (1981) 91-120

[3] Brezis H and Nirenberg L, Remarks on finding critical points, Comm. Pure Appl. Math. 44 (1991) 939-963

[4] Cac N P, On an elliptic boundary value problem at double resonance, J. Math. Anal. Appl. 132 (1988) 473-483

[5] Castro A and Cossio J, Multiple solutions for a nonlinear Dirichlet problem, SIAM J. Math. Anal. 25 (1994) 1554-1561

[6] Castro A, Cossio J and Neuberger J, On multiple solutions of a nonlinear Dirichlet problem, Nonlin. Anal. 30 (1997) 3657-3662

[7] Castro A and Lazer A, Critical point theory and the number of solutions of a nonlinear Dirichlet problem, Annali di Mat. Pura ed Appl. 70 (1979) 113-137

[8] Cerami G, Un criterio di esistenza per i punti critici su varietá illimitate, Istit. Lombardo Accad. Sci. Lett. Rend. A 112 (1978) 332-336

[9] Chang K C, Variational methods for nondifferentiable functionals and their applications to partial differential equations, J. Math. Anal. Appl. 80 (1981) 102-129

[10] Clarke F H, Optimization and nonsmooth analysis (New York: Wiley) (1983)

[11] Costa D and Silva E A, On a class of resonant problems at higher eigenvalues, Diff. Int. Eqns 8 (1995) 663-671

[12] Gasiński L and Papageorgiou N S, Solutions and multiple solutions for quasilinear hemivariational inequalities at resonance, Proc. R. Soc. Edinburgh 131A (2001) 1091-1111

[13] Gasiński L and Papageorgiou N S, An existence theorem for nonlinear hemivariational inequalities at resonance, Bull. Australian Math. Soc. 63 (2001) 1-14

[14] Gasiński L and Papageorgiou N S, Multiple solutions for semilinear hemivariational inequalities at resonance, Publ. Math. Debrecen 59 (2001) 121-146

[15] Goeleven D, Motreanu D and Panagiotopoulos P D, Eigenvalue problems for variational-hemivariational inequalities at resonance, Nonlin. Anal. 33 (1998) 161-180

[16] Hirano N and Nishimura T, Multiplicity results for semilinear elliptic problems at resonance with jumping nonlinearities, J. Math. Anal. Appl. 180 (1993) 566-586

[17] Hu S and Papageorgiou N S, Handbook of multivalued analysis, volume I: Theory (The Netherlands: Kluwer, Dordrecht) (1997)

[18] Hu S and Papageorgiou N S, Handbook of multivalued analysis, volume II: Applications (The Netherlands: Kluwer, Dordrecht) (2000) 
[19] Iannacci R and Nkashama M N, Nonlinear elliptic partial differential equations at resonance: Higher eigenvalues, Nonlin. Anal. 25 (1995) 455-471

[20] Jost J, Post-modern analysis (Berlin: Springer-Verlag) (1998)

[21] Kandilakis D, Kourogenis N and Papageorgiou N S, Two nontrivial critical points for nonsmooth functionals via local linking and applications, J. Global Optimization 34 (2006) 219-244

[22] Kourogenis N and Papageorgiou N S, Nonsmooth critical point theory and nonlinear elliptic equations at resonance, J. Austr. Math. Soc. 69A (2000) 245-271

[23] Landesman E, Robinson S and Rumbos A, Multiple solutions of semilinear elliptic problems at resonance, Nonlin. Anal. 24 (1995) 1049-1059

[24] Lebourg G, Valeur mayenne pour gradient génèralisé, CRAS Paris 281 (1975) 795-797

[25] Motreanu D and Panagiotopoulos P D, A minimax approach to the eigenvalue problem of hemivariational inequalities and applications, Applicable Anal. 58 (1995) 53-76

[26] Motreanu D and Panagiotopoulos P D, On the eigenvalue problem for hemivariational inequalities: Existence and multiplicity of solutions, J. Math. Anal. Appl. 197 (1996) 75-89

[27] Naniewicz Z and Panagiotopoulos P D, Mathematical theory of hemivariational inequalities and applications (New York: Marcel-Dekker) (1994)

[28] Niculescu C and Radulescu V, A saddle point theorem for nonsmooth functionals and problems at resonance, Annales Acad. Sci. Fennicae 21 (1996) 117-131

[29] Radulescu V, Mountain pass theorems for nondifferentiable functions and applications, Proc. Japan Acad. Sci. A69 (1993) 193-198

[30] Radulescu V and Panagiotopoulos P, Perturbations of hemivariational inequalities with constraints and applications, J. Global Optim. 12 (1998) 285-297

[31] Robinson S, Double resonance in semilinear elliptic boundary value problem over bounded and unbounded domains, Nonlin. Anal. 21 (1993) 407-424

[32] Su J, Semilinear elliptic boundary value problems with double resonance between two consecutive eigenvalues, Nonlin. Anal. 48 (2002) 881-895

[33] Su J and Tang C, Multiplicity results for semilinear elliptic equations with resonance at higher eigenvalues, Nonlin. Anal. 44 (2001) 311-321

[34] Tang C and Wu X P, Existence and multiplicity of semilinear elliptic equations, J. Math. Anal. Appl. 256 (2001) 1-12

[35] Tang C and Wu X P, Periodic solutions for second order systems with not uniformly coercive potential, J. Math. Anal. Appl. 259 (2001) 386-397

[36] Thews K, A reduction method for some nonlinear Dirichlet problems, Nonlin. Anal. 3 (1979) 795-813 